\title{
El valor científico de la Cueva-Mina Victoria (Cartagena, Murcia): un espacio donde el patrimonio natural y cultural se dan la mano
}

\begin{abstract}
Ainara Aberasturi ${ }^{a}$, Ignacio Fierro ${ }^{b}$, José Navarro ${ }^{a}$, Esther Bueno ${ }^{a}$, Gregorio Romero ${ }^{c}$ y Andrés Ros ${ }^{d}$
${ }^{a}$ Fundación Cidaris - Museo Paleontológico de Elche, Plaza San Juan s/n, 03203, Elche. ainara@cidarismpe.org, info@cidarismpe.org, ${ }^{\mathrm{b}} \mathrm{GeaLand}$ Patrimonio S.L., C/ Tibi no3 $3^{\mathrm{o}} \mathrm{E} 1$, 03010, Alicante. fierro@gealandpatrimonio.com, ${ }^{\mathrm{c} S e r v i c i o ~ d e}$ Patrimonio Histórico, Dirección General de Bienes Culturales, Consejería de Educación y Cultura, Casa Díaz-Casou, C/ Santa Teresa, 21, 30005, Murcia. gregorio.romero2@carm.es y ${ }^{\mathrm{d}}$ Ayuntamiento de Cartagena, C/ San Miguel, 8, 30201, Cartagena. aros@blog56.es.
\end{abstract}

\begin{abstract}
Resumen
La Cueva-Mina Victoria forma parte de un sistema cárstico de más de $3 \mathrm{~km}$ de longitud. Alberga un relleno Cuaternario con numerosos vertebrados, algunos descritos por primera vez. La actividad minera afectó a este enclave y permitió su descubrimiento, enriqueciendo al patrimonio natural con una historia y cultura singulares en la zona. Todo ello ha llevado a la protección legal del entorno como Bien de Interés Cultural. A pesar de esta riqueza en valores naturales y culturales, no se ha publicado, hasta la fecha, una valoración patrimonial del sitio que, de forma objetiva, exponga los criterios que aportan valor al bien y que permita un mínimo análisis o diagnóstico de los aspectos científicos más relevantes para su posterior gestión. Gracias a las ayudas económicas para la investigación e intervención del patrimonio arqueológico y paleontológico de la Región de Murcia y a la cofinanciación del Ayuntamiento de Cartagena, la Fundación Cidaris ha realizado, por primera vez, trabajos encaminados al conocimiento y gestión de los valores patrimoniales de la cueva. Se presentan los resultados del valor científico (Medio alto a Alto), discutiendo su significado y comparando los mismos con los de otros Lugares de Interés Geológico.
\end{abstract}

Palabras clave: Cartagena, Cueva Victoria, paleontología, patrimonio, valoración.

\begin{abstract}
Cave-Mine Victoria belongs to a more than $3 \mathrm{~km}$ long karst system. It holds a Quaternary fill highly rich in vertebrates, some of which were decribed there for the first time. Mining activity affected this site and allowed its discovery, thus enriching the natural heritage with the area unique history and culture. This has led to the legal protection of the site as Cultural Interest (BIC). Despite that natural and cultural richness, no heritage quantitative assessment publication has been done so far. There is a lack of publications objectively explaining the criteria that value the asset and allowing a minimum analysis or diagnosis of the more relevant scientific aspects in order to manage the site subsequently. Thanks to both, the region of Murcia economic aids for archaeological and palaeontological heritage research and intervention, and the joint financing from the City Council of Cartagena, the Cidaris Foundation has worked, for the first time, to enhance the knowledge and management of the cave heritage values. The results of the scientific assessment (Medium-high to High) are presented here, where its meaning is discussed and compared with other Spanish Geosites.
\end{abstract}

Keywords: Cartagena, Cueva Victoria, paleontology, heritage, assessment. 


\section{Introducción}

La Cueva-Mina Victoria (Cartagena, Región de Murcia) alberga un conocido yacimiento paleontológico que forma parte del Bien de Interés Cultural (BIC) incoado como "Sitio Histórico del Monte Miral" (Figs. 1 y 2). Sus valores geológicos y paleontológicos han propiciado su inclusión en el proyecto Global Geosite (IUGS y UNESCO). Además de este reconocimiento y figura de protección, el yacimiento paleontológico se encuentra recogido en diferentes inventarios de ámbito local, regional y nacional.

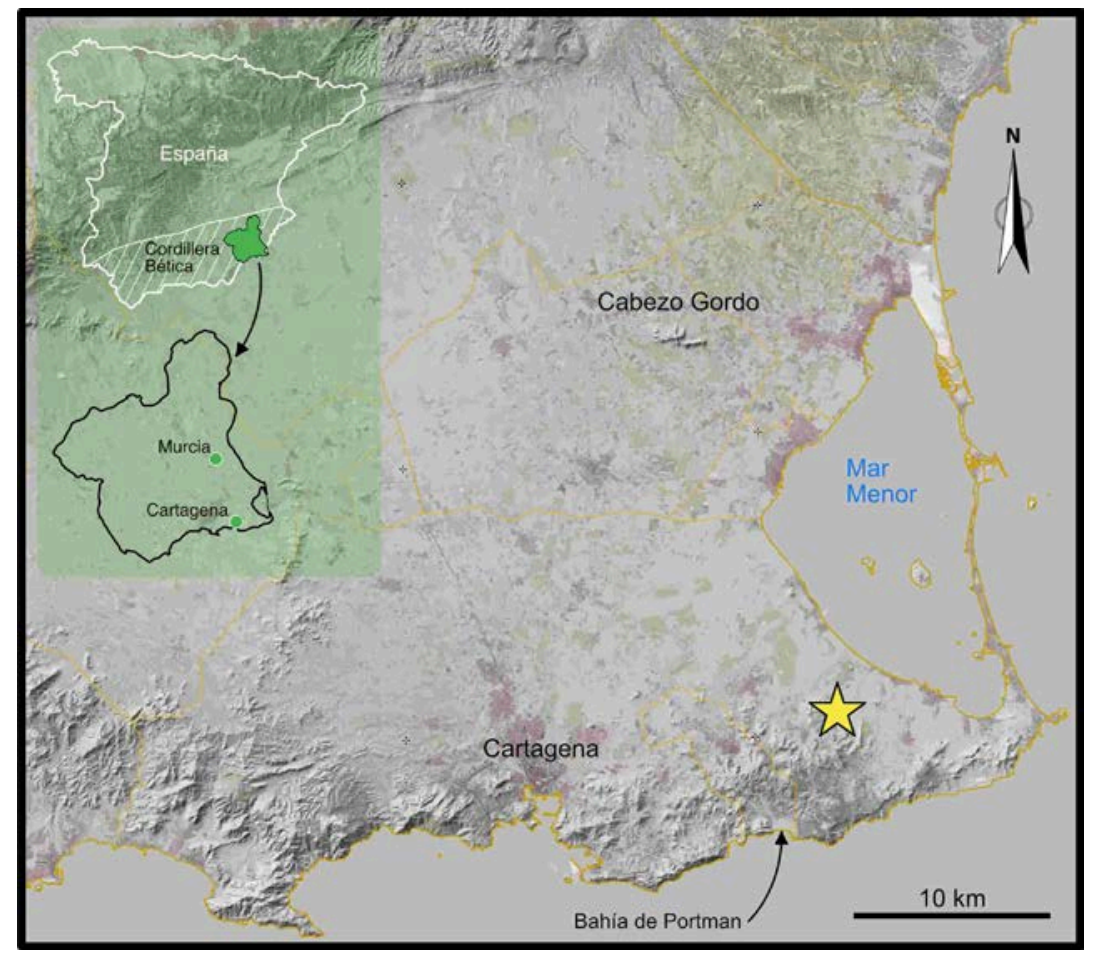

Fig. 1 Localización general de la Cueva-Mina Victoria (estrella amarilla), al sur del Mar Menor

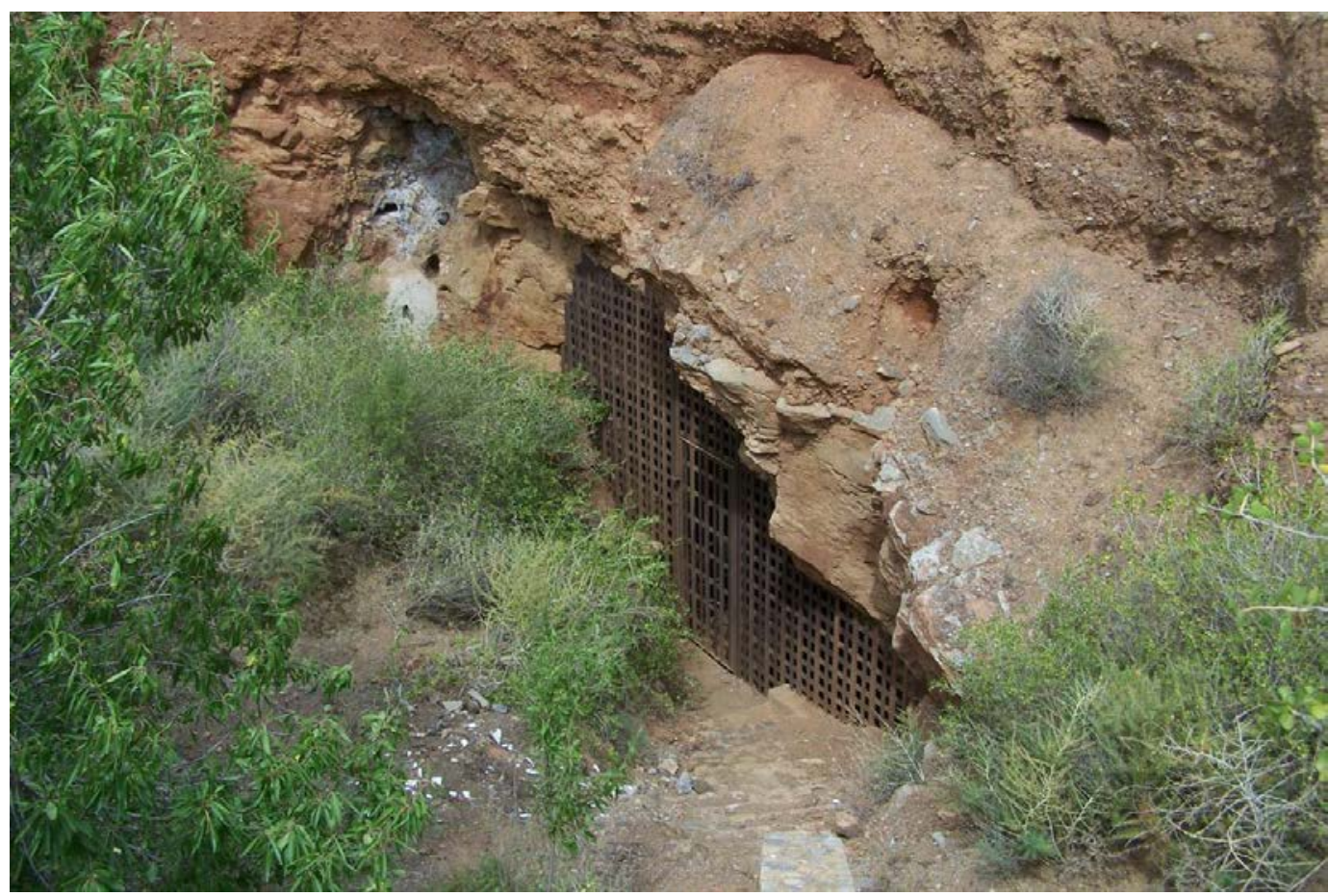

Fig. 2 Vista de una de las entradas al yacimiento (Victoria I) 
El yacimiento se dió a conocer en el año 1970, realizándose posteriormente diferentes intervenciones esporádicas lideradas por Juan Pons Moya en las que se recupera fauna fósil (Pons-Moyà y Moyà-Solà, 1978). La identificación de una falange humana (Gibert y Pons-Moyà, 1984) propicia el inicio de un proyecto de investigación dirigido por el Dr. J. Gibert hasta el año 2007, cuando falleció poco después de terminar la campaña de excavaciones. Finalmente dos colaboradores de J. Gibert (C. Ferràndez y L. Gibert) deciden actualizar todo el conocimiento disponible sobre la cavidad. Así, se realiza una exposición sobre el yacimiento, "Cueva Victoria - Out of Africa”, junto a la publicación de una monografía (Mastia, 2015 vol. 11-12-13) y varias publicaciones científicas en revistas internacionales (p. ej. Gibert et al., 2015).

El elevado grado de conocimiento de este enclave y la posibilidad de musealizar el mismo, estableciendo una oferta de ocio y turismo en la zona han llevado al desarrollo del reciente borrador del Plan Director de la cueva (Laine et al., 2018). A pesar de todo lo anterior, la Cueva-Mina Victoria carece de una valoración patrimonial que haya sido objeto de publicación o debate.

Entre julio y agosto de 2019 un equipo de paleontólogos de la Fundación Cidaris lideró un campo de trabajo en Cueva Victoria que perseguía diferentes objetivos relacionados con el patrimonio geológico y paleontológico, entre ellos, un análisis objetivo del valor científico de la cueva así como el estudio de su potencialidad de uso (Fig. 3).

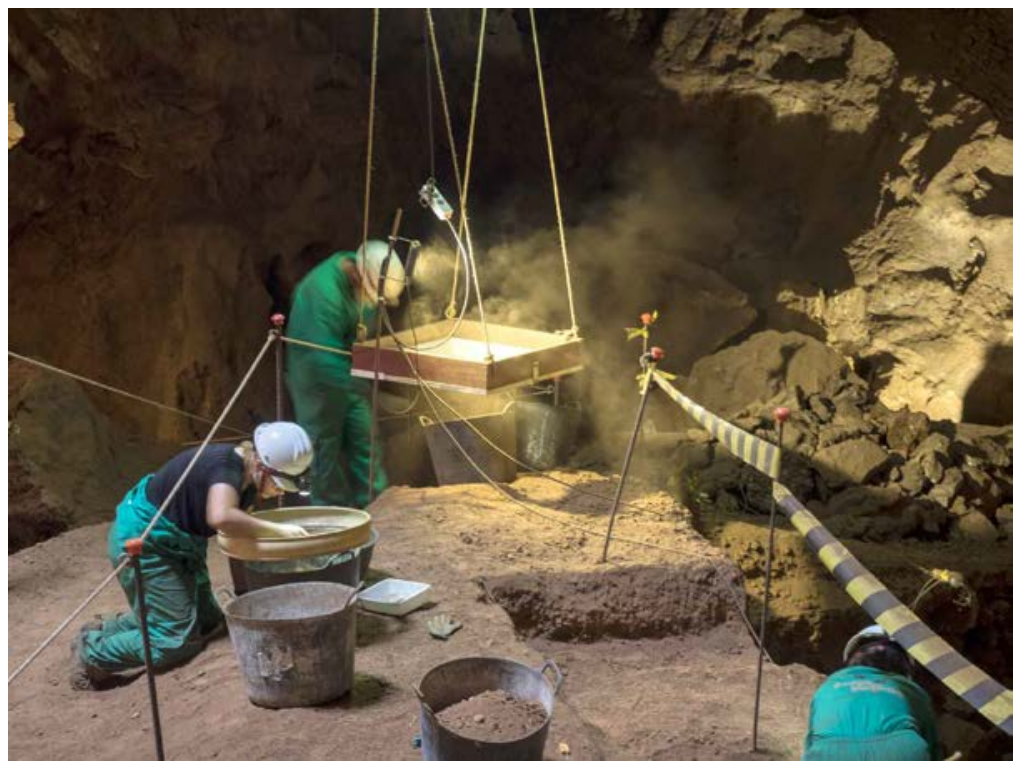

Fuente: Andrés Ros - Fundación Cidaris

Fig. 3 Tareas de tamizado durante el campo de trabajo-formación desarrollado en verano de 2019. Cuadrícula en Sondeo 3

Este trabajo tiene como objetivos la valoración del interés científico o intrínseco de la cueva-mina y su comparación con resultados previamente existentes. Además, se persigue un análisis comparativo con el valor otorgado a otros yacimientos paleontológicos o lugares de interés geológico del entorno.

\section{Metodología}

El cálculo del interés científico de un enclave de naturaleza geológica supone una parte muy concreta de su estudio patrimonial, pero es la base para el inicio de su gestión. Desde el año 2006 un equipo multidisciplinar del Museo Paleontológico de Elche (MUPE) y de la Fundación Cidaris trabajan en un modelo de gestión del patrimonio geológico que tiene como base la valoración patrimonial (FOPALI, Fósiles y Patrimonio de Alicante).

En la valoración científica de FOPALI se emplean un total de 10 criterios: Abundancia de afloramientos similares, Localidad tipo, Paleodiversidad, Paleodisparidad, Estado de conservación inmueble, Estado de conservación mueble, Interés tafonómico, Interés geológico, Utilidad para ilustrar procesos y Potencial científico. Para una información detallada del modelo puede consultarse la referencia original en Sánchez et al. (2019). Todos estos criterios tienen igual 
peso o relevancia y se bareman con puntuaciones de 0 a 4 . El modelo permite el cálculo de un valor final al realizar una media aritmética, pudiendo ofrecerse el resultado sobre un valor máximo de 4, normalizado a un valor máximo de $10 \mathrm{o}$ en porcentaje. A pesar de ser un modelo semicuantitativo, su aplicación se basa en un sistema de subjetividad compartida en el que un equipo multidisciplinar discute hasta consensuar el valor otorgado a cada criterio.

\section{Resultados: valor científico}

\subsection{Abundacia de afloramientos similares (1)}

Cueva Victoria es un yacimiento paleontológico con restos de vertebrados del Pleistoceno inferior. Se contextualiza asociado a un relleno cárstico en el Cerro de San Ginés, espacio que posteriormente fue afectado por el desarrollo minero en la zona. Es el único yacimiento del Pleistoceno inferior en un relleno cárstico del término municipal de Cartagena, no así en la Región de Murcia, donde encontramos otro yacimiento con las mismas características: el de la Sierra de Quibas (Abanilla) (Montoya et al., 1999). Por otro lado, el yacimiento de Cueva Negra (Caravaca de la Cruz), se situa cronológicamente en la transición del Pleistoceno inferior al Pleistoceno Medio (Walker et al., 2013), aunque su relleno posiblemente se relacione con inundaciones fluviales periódicas y no tanto con el origen del carst. La distancia de un yacimiento a otro es entre 75 y $100 \mathrm{~km}$, teniendo la Comunidad Autónoma tres afloramientos en rellenos cársticos con fósiles del Pleistoceno inferior.

Por ello, según el modelo de valoración de FOPALI, al ser el yacimiento único en el término municipal pero no a nivel de Comunidad Autónoma (y por tanto, tampoco a nivel nacional o mundial), se le asigna a esta categoría un valor de 1.

\subsection{Localidad tipo (4)}

Los restos fósiles recuperados en Cueva Victoria son de gran interés ya que, entre ellos, se han hallado varias especies y un género desconocidos previamente. Así, los taxones definidos en el yacimiento son 4: el cánido Cuon rosi (PonsMoyà y Moyà-Solà, 1978), el lagomorfo Oryctolagus giberti (De Marfà, 2008), el cérvido Megaloceros novocarthaginiensis (Van der Made, 2015), y el arvicólido Allophaiomys chalinei (Alcalde et al., 1981), que pasó a ser la especie tipo de un nuevo género, Victoriamys (Martin, 2012).

Hasta la fecha, Cueva Victoria es la localidad tipo de tres especies y un género. El modelo de valoración FOPALI da más importancia a las categorías taxonómicas más inclusivas, dando el valor máximo (4) para definir un yacimiento como localidad tipo a nivel de género, en nuestro caso de Victoriamys.

\subsection{Paleodiversidad (4)}

En el yacimiento de Cueva Victoria, antiguamente un cubil de hienas, se han encontrado numerosas especies que vivieron en la cueva, sus alrededores o fueron depredadas y transportadas por estas hienas. De acuerdo con la asociación faunística de Cueva Victoria recogida en Fernàndez-Cañadell y Gibert (2015), se ha confirmado la presencia de una gran cantidad de especies diferentes, que se incluyen en diversos grupos: 1 gasterópodo, 6 anfibios, 8 escamosos, 1 testudínido, 44 aves, 2 roedores, 3 lagomorfos, 6 quirópteros, 2 insectívoros, 3 perisodáctilos, 4 bóvidos, 2 cérvidos, 1 proboscídeo, 5 felidos, 1 úrsido, 1 hiénido, 2 cánidos, 1 fócido, 1 cetáceo y 2 primates.

El número total de especies identificadas es de 96 , dato que está muy por encima de 20, el requerido para otorgar al yacimiento la puntuación máxima (4) en Paleodiversidad.

\subsection{Paleodisparidad (1)}

A pesar de rondar el centenar de especies, el yacimiento de Cueva Victoria es poco dispar a nivel de filos, pues solo hay representantes de 2 de ellos. De hecho, la práctica mayoría de las especies encontradas pertenecen al filo Chordata, excepto una de ellas, el gasterópodo, que pertenece al filo Mollusca.

El modelo FOPALI otorga un valor de 1 en el criterio de Paleodisparidad a yacimientos que, como en este caso, presentan organismos de 2 filos diferentes. 


\subsection{Estado de conservación inmueble (2)}

Cueva Victoria se descubrió gracias a la actividad minera. Sin embargo, dicha actividad en Mina Victoria, que duró alrededor de 60 años, deterioró parte de la cueva natural (Pérez de Perceval et al., 2015). Aunque la minería hizo la cueva accesible y permitió la posterior excavación del yacimiento, afectó a todos los elementos naturales de su interior. La mayor parte de la brecha cárstica fue extraída durante esta actividad. Sin embargo, actualmente, en el inmueble se pueden apreciar sus características clave, permitiendo su investigación y la delimitación y estudio de varias localidades en las que se recuperan restos fósiles (Fernàndez-Cañadell y Gibert, 2015).

El estado de conservación de algunas de estas localidades (Andamio A y B, Sondeo 3 y los bloques caídos de Sala La Unión o Sala José Gibert) ha podido ser valorado durante el desarrollo del campo de trabajo efectuadado por la Fundación Cidaris en 2019. Efectivamente, el inmueble se encuentra afectado; Andamio A y B, constituyen los últimos restos de la brecha adosada a la pared de la cavidad, Sondeo 3 es una escombrera minera con restos de vertebrados de origen desconocido y los bloques caídos en Sala José Gibert han sido afectados por la caída de los mismos y por la extracción de huesos mediante técnicas poco apropiadas.

Con todo esto, podemos concluir que el inmueble se encuentra afectado pero en el se aprecian características clave que permiten profundizar en su conocimiento (valor 2 en el modelo). De hecho, ha sido posible realizar estudios del inmueble en relación a sus fósiles, como el modelo de génesis de acumulación osífera de Vilà-Vinyet et al. (2015), donde se utiliza la orientación de los mismos.

\subsection{Estado de conservación mueble (4)}

Se pueden observar diferentes estados de conservación en los fósiles extraídos de Cueva Victoria; esto se debe a los procesos sufridos por los restos durante el transporte, la compactación de los sedimentos y las condiciones de la cueva. Sin embargo, a pesar de estar afectados por factores tafonómicos o fosildiagenéticos (Fernàndez-Cañadell, 2015), su estado de conservación es muy bueno, incluso para los fósiles más pequeños.

La preservación de estos fósiles ha facilitado su estudio y ha permitido, como ya se ha señalado, la identificación de 96 especies diferentes, muchas de las cuales pertenecen a microvertebrados. Esta conservación se considera como "muy buena" siguiendo el modelo FOPALI y tiene un valor de 4 (el máximo) en este parámetro.

\subsection{Interés tafonómico y genético (4)}

Cueva Victoria permite establecer una secuencia de procesos geomorfológicos a partir de su formación y de las fases en su relleno. La génesis de la cueva es particular (Fernàndez-Cañadell, 2015; Ros y Llamusí, 2015), ya que resulta ser de origen hipogénico, relacionada con la acidificación de las aguas debido a la presencia próxima de sulfuros, fruto de procesos hidrotermales derivados de la actividad volcánica que produjo las mineralizaciones de la Sierra Minera. Al abrirse al exterior, por disolución y colapso, empezaría el proceso de relleno de la cueva, colmatándola con alóctonos provenientes de los abanicos aluviales del Cerro de San Ginés. En ese momento empiezan la acumulación de fósiles, destacando el importante papel del agua (entrada natural) y las hienas (entrada forzada) en la restos. El relleno fue rápido y no presenta discontinuidades. Durante la posterior compactación y cementación del relleno se generó una colada espeleotémica (flowstone) que lo recubre, correlacionable en toda la cavidad.

Con respecto al interés tafonómico tenemos por un lado restos de microvertebrados y hiénidos, como habitantes de la cueva y, por otro, macrovertebrados transportados por la acción de estas últimas (algunos con marcas de depredación) o bien introducidos en la cueva por alguna fisura. También se encuentran acumulaciones de coprolitos de hiena. La acumulación de restos fósiles estaba en parte condicionada por corrientes direccionales que se distribuían a partir de un cono de deyección situado en la entrada original de la cueva (Vilà-Vinyet et al., 2015).

Tanto la tafonomía como la génesis de la cavidad están rodeadas de elementos particulares que resultan de gran interés, por lo que este criterio se valora como "muy alto" (4) en el modelo FOPALI. 


\subsection{Interés geológico $(1,50=1)$}

El valor total del interés geológico se debe a la media redondeada a la unidad de la puntuación de los siguientes parámetros:

\subsubsection{Bioestratigrafia (1)}

Cueva Victoria, con más de 70 especies de vertebrados, presenta especies de mamíferos con significado bioestratigráfico (Gibert y Scott, 2015). Sin embargo, la potencia de los depósitos y el conjunto de la fauna no permite el establecimiento de biozonas. Los restos fósiles de mayor interés a este respecto suelen ser los micromamíferos de los que tan solo se han identificado cuatro especies, solo una con cierto valor bioestratigráfico. En definitiva, estos restos solo permiten acotar una edad entre los 1,3 y los 0,7 Ma, pero no son decisivos para el establecimiento de la edad ni caracterizan al cuerpo rocoso asociándolo a una edad concreta. Por este motivo consideramos que el valor bioestratigráfico es bajo (1).

\subsubsection{Litoestratigrafia (1)}

La secuencia estratigráfica del relleno de Cueva Victoria es bien conocida y consta de dos unidades litoestratigráficas (Ferràndez et al., 1989): una unidad inferior de arcillas rojas y una unidad superior con la brecha fosilífera que se encuentra cubierta con una espeleotema. Esta secuencia es de gran interés pues registra el proceso de disolución de la cueva y el relleno de la misma en su momento de conexión con el exterior. La utilidad de este registro es muy local, no ha permitido la definición de formaciones y su correlación tan solo es útil en el interior de la propia cueva. Por todo ello, el valor que asignamos a este criterio es bajo (1).

\subsubsection{Tectónica (1)}

Desde el punto de vista tectónico la Cueva-Mina Victoria presenta cierta relación genética con la tectónica local. La mineralización primaria se encuentra asociada a una red de fracturas $\left(\mathrm{N} 130^{\circ} \mathrm{E}\right.$ y $\left.\mathrm{N} 20^{\circ} \mathrm{E}\right)$ en las calizas del Cabezo de San Ginés. La presencia de la falla de Cartagena-El Estrecho (Manteca y Ovejero, 1992), al sur del cabezo, ha jugado un papel importante al posicionar el manto piritoso de Los Blancos a cotas que han permitido una posible liberación ácida y la aceleración del proceso cárstico. Más allá de esta singularidad geológica relacionada con la tectónica, la cueva-mina no presenta un mayor interés tectónico, motivo por el que ofrecemos un valor bajo (1) a este criterio.

\subsubsection{Geomorfología (3)}

Desde el punto de vista geomorfológico, existen múltiples elementos vinculados al origen de la cueva (procesos cársticos) y al relleno de la misma, incluyendo la singularidad paleontológica, que son de interés y han suscitado su investigación. Las diferentes morfologías de disolución y las espeleotemas desarrolladas apuntan al origen hipogénico de la cavidad, unido a un carst epigénico más reciente (Ros y Llamusí, 2015). El desarrollo posterior de un relleno del Pleistoceno inferior (con fósiles) y otro más moderno de depósitos de ladera cementados (escasamente estudiado), en cuyo depósito juega un papel importante el agua y la forma que tendría el Cerro de San Ginés y las zonas de entrada a la cueva, son elementos de gran interés geomorfológico. Su estudio puede servir para modelizar la posición de otras zonas en las que puedan existir más acumulaciones óseas. Por último, en el interior de la actual cavidad existe una gran mezcla, con grandes bloques caídos de los techos y paredes que poseen restos fósiles. Su procedencia es digna de ser estudiada, pues de algunos de ellos se han extraído elementos fósiles importantes. En base a todo lo anterior, podemos indicar que el valor geomorfológico del lugar es alto (valor 3).

\subsection{Utilidad para ilustrar procesos (4)}

Cueva Victoria ofrece un marco incomparable para ilustrar y poder estudiar múltiples procesos, entre los que cabe destacar la paleoclimatología, pues los espeleotemas actúan como registros o bases de datos de la información climática del pasado. El registro espeleotémico de la cueva ha sido objeto de estudios en esta área, como los de Budsky et al. $(2015 ; 2019)$. 
Otro de los procesos que pueden analizarse en la cueva es la propia génesis del carst (Ros y Llamusí, 2015) o del contexto sedimentario de la cavidad y su relleno (Fernàndez-Cañadell, 2015). Asimismo, Cueva Victoria resulta un ejemplo para ilustrar la fauna del Pleistoceno inferior ibérico, pues se encuentran numerosas especies de esta época e incluso de ambientes diferentes (Fernàndez-Cañadell y Gibert, 2015).

Todo ello hace que la valoración para este parámetro en Cueva Victoria sea el máximo (valor 4), pues son múltiples los procesos que han sido estudiados en ella y que pueden ser explicados de forma independiente utilizando el registro de la cueva.

\subsection{Potencial científico (2)}

El yacimiento paleontológico de Cueva Victoria fue dado a conocer a la comunidad científica en 1970 y, a partir de ese momento, ha sido objeto de numerosas publicaciones que se han realizado de manera discontinúa en el tiempo hasta la actualidad. En este sentido, destacan dos momentos bien marcados: uno correspondiente a 1990 en el que se produce un aumento muy significativo de los artículos publicados y otro entre 2012-2014 que corresponde con la publicación de la monografía Mastia (Eds.: Gibert y Ferràndez-Cañadell, 2015). Así, un tercio de las publicacones del yacimiento son de este último periodo, por lo que gran parte del conocimiento de la cavidad se ha publicado recientemente.

En base al grado de conocimiento existente y al volumen de elementos de interés científico todavía presentes en la cueva, consideramos que el potencial científico de este enclave es medio (valor 2 según el modelo). Algunos elementos de gran interés que pueden permitir seguir investigando en la cueva son: la propia brecha osífera y las espeleotemas y su relación con el clima del Cuaternario. Finalmente, desde nuestro punto de vista, una de las líneas de investigación con mayor potencial en este momento de conocimiento de la cueva se relaciona precisamente con el patrimonio y su gestión. En este sentido, este trabajo, destinado a conocer el interés científico de la cueva, es uno de los pioneros y abre un campo potencial en el que esta línea de investigación se vuelve esencial para la correcta gestión de dicho enclave y su potencial científico.

\section{Discusión y conclusiones}

En base a los resultados de los criterios de valoración previamente expuestos, hemos obtenido un valor científico para Cueva Victoria de 27 puntos sobre un máximo de 40, siendo equivalente a un 67,50 \% (6,75 sobre 10) (tabla 1). Según el modelo FOPALI, estos datos son indicativos de un valor Medio alto a Alto, más próximo a este último.

Tabla 1. Valor científico final del yacimiento de Cueva Victoria usando el modelo FOPALI

\begin{tabular}{|c|c|c|c|}
\hline \multicolumn{4}{|c|}{ VALORACIÓN CIENTÍFICA CUEVA VICTORIA MODELO FOPALI } \\
\hline Parámetro & Puntos & Valor medio (0-4) & Valor científico (0-10) \\
\hline Abundancia de afloramientos similares & 1 & \multirow{10}{*}{2,7} & \multirow{10}{*}{6,75} \\
\hline Localidad tipo & 4 & & \\
\hline Paleodiversidad /Geodiversidad & 4 & & \\
\hline Paleodisparidad & 1 & & \\
\hline Conservación inmueble & 2 & & \\
\hline Conservación mueble & 4 & & \\
\hline Interés tafonómico/genético & 4 & & \\
\hline Interés geológico & 1 & & \\
\hline Utilidad como modelo de procesos & 4 & & \\
\hline Potencial científico & 2 & & \\
\hline TOTAL & 27 & & \\
\hline
\end{tabular}

Este dato contrasta con el único valor que existe publicado de este sitio, siguiendo el modelo del Inventario Español de Lugares de Interés Geológico (IELIG), donde se da la cifra de 4,1 sobre 10 (Jordá, 2019). A pesar de la diferencia numérica, siguiendo la metodología del IELIG este valor también es considerado Alto por encontrarse en el rango 3,336,65. Podemos concluir que, a pesar de la gran diferencia existente en los resultados cuantitativos de las valoraciones con los modelos FOPALI y IELIG, la categorización cualitativa de los mismos es similar: el valor científico es Alto. 
Por otro lado, queremos destacar la utilidad de la valoración patrimonial como herramienta para justificar objetivamente el interés intrínseco de un bien y, por lo tanto, su necesidad de protección. Con demasiada frecuencia la aplicación de una protección legal a un bien geológico se desvincula de su valor científico o, este, se argumenta laxamente. Hemos realizado una comparativa entre el valor de 4 yacimientos paleontológicos ( 2 de Alicante y 2 de la Región de Murcia) y su estatus de protección legal (Fig. 4).

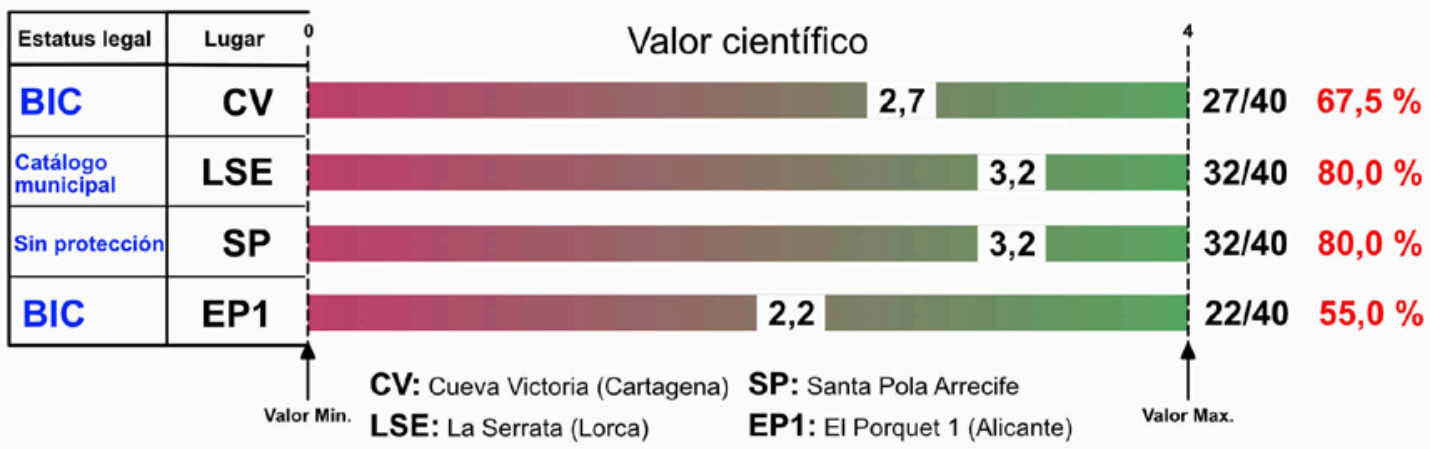

Fuente: Sánchez et al. (2019)

Fig. 4 Valor científico de diferentes enclaves geológicos (Alicante y Murcia). Datos derivados de la aplicación del modelo FOPALI

De los datos presentados se deriva la existencia de importantes yacimientos (La Serrata en Lorca o el arrecife fósil de Santa Pola) que a pesar de su enorme interés científico no gozan de la máxima figura de protección (BIC); en el caso de Santa Pola no existe protección alguna. Por otro lado, yacimientos paleontológicos como Cueva Victoria (Cartagena) y El Porquet 1 (Alicante), con valores inferiores o muy inferiores, respectivamente, han sido declarados BIC por las administraciones competentes. Nos parece necesario que en un futuro se vincule la estrategia de gestión de estos enclaves, poseedores de valores culturales y naturales, a una herramienta de valoración patrimonial.

Finalmente, tal y como se deriva de la "Carta ICOMOS para la Interpretación y Presentación de Sitios de Patrimonio Cultural”, la transferencia social de los valores científicos de Cueva Victoria debe ser abordada en el futuro. Debemos empezar por realizar una valoración de la potencialidad de uso de este enclave que, de forma rigurosa y respetando los valores científicos, permita presentar en qué situación se encuentra el posible uso social de este magnífico yacimiento. Para ello, los principios fundamentales recogidos en la Carta nos parecen esenciales, destacando la sostenibilidad, la participación e inclusión y la investigación como actividad continua a desarrollar.

\section{Referencias}

Alcalde, G., Agustí, J., y Villalta, J. F. (1981). Un nuevo Allophaiomys (Arvicolidae, Rodentia, Mammalia) en el Pleistoceno Inferior del sur de España. Acta Geológica Hispánica, 16, 203-205.

Budsky, A., Scholz, D., Gibert, L., y Mertz-Kraus, R. (2015). 230Th/U-dating of the Cueva Victoria flowstone sequence: Preliminary results and paleoclimate implications. Mastia: Revista del Museo Arqueológico Municipal de Cartagena, 11-12-13, 101-109.

Budsky, A., Wasswenburg, J. A., Mertz-Kraus, R., Spötl, C., Jochum, K. P., Gibert, L. y Scholz, D. (2019). Western Mediterranean climate response to Dansgaard/Oeschger Events: New insights from speleothem records. Geophysical Research Letters, 46, 9042-9053.

De Marfà, R. (2008). Oryctolagus giberti n. sp. (Lagomorpha, Mammalia) du Pléistocène inférieur de Cueva Victoria (Murcia, Espagne). C. R. Palevol, 7, 305-313.

Fernàndez-Cañadell, C. F. (2015). Contexto sedimentario y Tafonomía del yacimiento de Cueva Victoria. Mastia: Revista del Museo Arqueológico Municipal de Cartagena, 11-12-13, 139-162.

Fernàndez-Cañadell, C. F., y Gibert, L. (2015). Introducción. Cueva Victoria, un yacimiento de vertebrados del Pleistoceno Inferior. Mastia: Revista del Museo Arqueológico Municipal de Cartagena, 11-12-13, 17-45. 
Ferràndez, C., Pérez-Cuadrado, J. L., Gibert, J., y Martínez, B. (1989). Estudio preliminar de los sedimentos de relleno de Cueva Victoria (Cartagena, Murcia). En J. Gibert, D. Campillo, y E. García Olivares (Eds.), Los Restos Humanos de Orce y Cueva Victoria (pp. 379-393). Barcelona: Publicacions de l'Institut de Paleontologia Dr. M. Crusafont.

Gibert, L., y Ferràndez-Cañadell, C. (Eds.). (2015). Geología y Paleontología de Cueva Victoria. Mastia: Revista del Museo Arqueológico Municipal de Cartagena, 11-13.

Gibert, J., y Pons-Moyà, J. (1984). Estudio morfológico de la falange del género Homo de Cueva Victoria (Cartagena, Murcia). Paleontologia i Evolucio, 18, 49-55.

Gibert, L., Scott, G. R., Scholz, D., Budsky, A., Ferràndez, C., Ribot, F., Martin, R. A. y Lería, M. (2015). Chronology for the Cueva Victoria fossil site (SE Spain): Evidence for Early Pleistocene Afro-Iberian dispersals. Journal of Human Evolution, 90, $183-197$.

Jordá Pardo, J. F. (2019). BE004. Cueva Victoria. Ficha oficial del LIG asociada al IELIG. Instituto Geológico y Minero de España.

Manteca Martínez, J. I., y Ovejero Zappino, G. (1992). Los yacimientos Zn, Pb, Ag-Fe del distrito minero de La Unión-Cartagena, Bética Oriental. En Recursos Minerales de España (pp. 1085-1102). Madrid: CSIC.

Martin, R. A. (2012). Victoriamys, a new generic name for Chaline's vole from the Pleistocene of Western Europe. Geobios, 45, $445-450$

Montoya, P., Alberdi, M. T., Blázquez, A. M., Barbadillo, L. J., Fumanal, M. P., van der Made, J., Marín, M., Molina, A., Morales, J., Murelaga, X., Peñalver, E., Robles, F., Ruiz Bustos, A., Sánchez, A., Sanchiz, B., Soria, S., y Szyndlar, Z. (1999). La fauna del Pleistoceno Inferior de la Sierra de Quibas (Abanilla, Murcia). Estudios geológicos, 55:3-4, 127-161.

Pérez de Perceval, M. A., Manteca Martínez, J. I., y López-Morell, M. A. (2012-14). Historia de la minería de Cueva Victoria. Mastia: Revista del Museo Arqueológico Municipal de Cartagena, 11-12-13, 47-58.

Pons-Moyà, J. y Moyà-Solà, S. (1978). La fauna de Carnívoros del Pleistoceno medio (Mindel) de la Cueva Victoria (Cartagena, España). Acta Geologica Hispanica, 13, 54-58.

Ros, A., y Llamusí, J.L. (2012-14). Reconstrucción y génesis del karst de Cueva Victoria. En Mastia: Revista del Museo Arqueológico Municipal de Cartagena, 11-12-13, 111-125.

Sánchez Ferris, J. E., Fierro Bandera, I., Aberasturi Rodríguez, A., Navarro Pedreño, J., y Montoya Belló, P. (2019). La valoración del patrimonio geológico y paleontológico como herramienta de gestión: el modelo FOPALI. Spanish Journal of Palaeontology, $34: 1,35-56$.

Van der Made, J. (2012-14). The latest Early Pleistocene giant deer Megaloceros novocarthaginiensis n. sp. and the fallow deer" Dama cf. vallonnetensis" from Cueva Victoria (Murcia, Spain). Mastia: Revista del Museo Arqueológico Municipal de Cartagena, 11-12-13, 269-323.

Vilà-Vinyet, J., Soriguera-Gellida, I., y Fernàndez-Cañadell, C. (2015). Génesis de una acumulación osífera excepcional en Cueva Victoria (Cartagena, Murcia, España). Mastia: Revista del Museo Arqueológico Municipal de Cartagena, 11-12-13, 163-173.

Walker, M. J., López-Martínez, M., Carrión-García, J. S., Rodríguez-Estrella, T., Del-Toro, M. S. N., Schwenninger, J. L., LópezJiménez, A., Ortega-Rodrigáñez, J., Haber-Uriarte, M., Polo-Camacho, J. L., García-Torres, J., Campillo-Boj, M., AvilésFernández, A., y Zack, W. (2013). Cueva Negra del Estrecho del Río Quípar (Murcia, Spain): A late Early Pleistocene hominin site with an "Acheulo-Levalloiso-Mousteroid" Palaeolithic assemblage. Quaternary International, 294, 135-159. 